\title{
Towards Cross-Lingual Generalization of Translation Gender Bias
}

\author{
Won Ik Cho* \\ Dept. of ECE and INMC \\ Seoul National University \\ Seoul, Korea \\ wicho@hi.snu.ac.kr \\ Jaeyeong Yang \\ Dept. of Linguistics \\ Seoul National University \\ Seoul, Korea \\ tastymango@snu.ac.kr
}

\author{
Jiwon Kim*
Independent Researcher
Daegu, Korea
kimjiwon08@snu.ac.kr \\ Jiwon Kim*
Independent Researcher
Daegu, Korea
kimjiwon08@snu.ac.kr \\ Jiwon Kim*
Independent Researcher
Daegu, Korea
kimjiwon08@snu.ac.kr \\ Jiwon Kim*
Independent Researcher
Daegu, Korea
kimjiwon08@snu.ac.kr
}

Nam Soo Kim

Dept. of ECE and INMC

Seoul National University

Seoul, Korea

nkim@snu.ac.kr

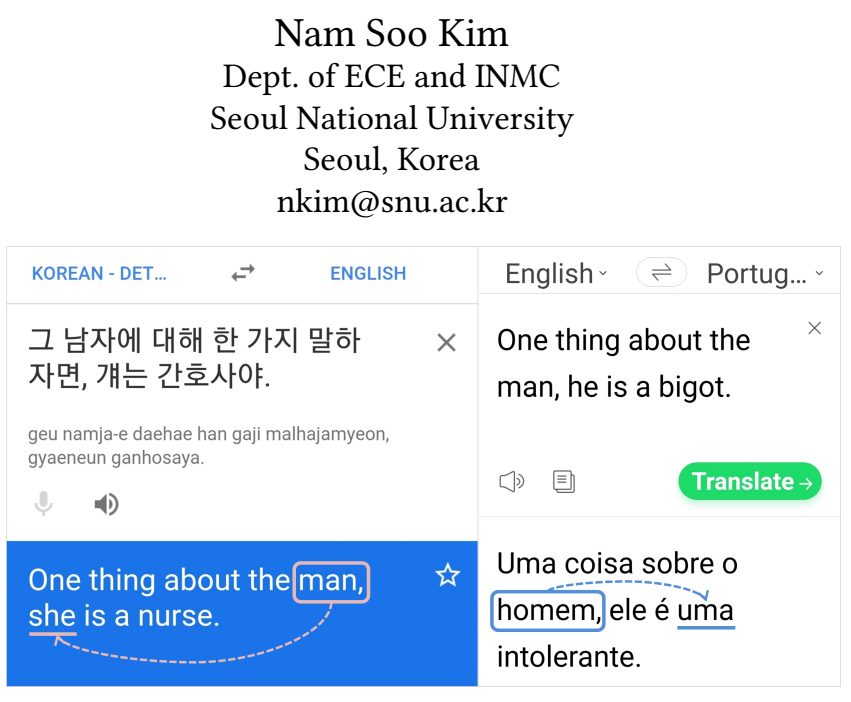

Figure 1: Outputs of publicly available machine translation services incorporating the social bias, observable by a pronoun (left) and the feminine article uma (right). Both cases yield wrong results, even though given a clue to the gender in the preceding clause.

Virtual Event, Canada. ACM, New York, NY, USA, 9 pages. https://doi.org/ $10.1145 / 3442188.3445907$

\section{INTRODUCTION}

Research on the detection and mitigation of social bias in artificial intelligence (AI) models is not only of social concerns but also an engineering issue that should be resolved regarding the accuracy of models. Natural language processing (NLP) is the representative area where the injection of bias is visible, since its main ingredient, corpus, incorporates various social concepts that largely influence the inductive learning procedure of machines. Among such phenomena, the gender bias projected during automatic machine translation (MT) (Figure 1) is one of the most significant that its identification $[17,22,26]$ and resolution $[10,23]$ have been discussed in many regards. While dealing with the definition of the problem [14], formulation of the template [20], and measurement [6], recent approaches made an expansion to both genealogically and typologically diverse languages rather than just concentrating on Germanic, Romance, or other Indo-European languages.

So far, however, few papers took a cross-lingual stance, where different types of gender bias in the translation are integrated into 
the measure. For example, the aspect of bias observed in KoreanEnglish translation differs from that of English-Portuguese (Figure 1); thus, the template and the measurement must accommodate this diversity. Here, we tackle the lack of this kind of approach by selecting the source or target languages based on various linguistic viewpoints, generating a set of template sentences considering various factors such as occupations, sentiment words, and contexts for coreference. Our contribution to this field can be summarized as follows:

- Criteria on grouping the languages on the basis of common characteristics, making the investigation of the translation gender bias more typological and cross-linguistically generalizable

- A new template and content word-based evaluation that can be used to measure the bias across different types of language pairs

As a core motivation of our research, we focus on the direct harms to the end-users of public MT systems that call for considerations in ethics and multilingualism. It is prevalent that the users face biased and offensive output, which might induce incorrectness and harassment even if one is not familiar with either the source or target language. We aim to expand the preceding analyses to see how the tendency differs by language typology and resource. For further description and justification, we shall provide a brief summary of the literature on bias in AI and NLP.

\section{RELATED WORK}

Ever since Friedman and Nissenbaum [11] gave a definition of computer system bias, researchers have examined stereotypes thereof, its influence on society, and how to remove it. These studies focused on traditional computer systems, which worked differently from nowadays. In that regard, Binns [2] elucidated emerging arguments on machine learning bias, which increasingly affects people and society.

One pioneer work on gender issues deals with image semantic role labeling, mainly on AIs' prejudice in comprehending pictures depicting real-life topics [30]. The paper attacks the biased inference of image comprehension tasks, such as describing the man cooking in the kitchen as a woman cooking if the kitchen interior is bright and fancy. Also, the paper aggregates gender-related constraints in the training phase for the mitigation.

Alongside such visual factors, NLP takes up a significant portion of recent machine learning studies. Though not as visible and intuitive as in the vision area, people using various NLP applications face explicit or implicit types of bias as well.

Accordingly, in purely text-based settings, many studies centered on evaluating the biasedness of gender-context correlation throughout the corpus [16] and mitigating it by, e.g., swapping the gender [21]. Especially, in the context of widely used pre-trained language models, Bolukbasi et al. [4] shed light on the word relation test, revealing phenomena such as man: computer programmer $\approx$ woman : homemaker. Up-to-date studies claimed that such kind of experiment could not represent the bias in its entirety [18], but the tendency is still preserved in that the learning-based NLP frameworks integrate corpus-level bias projected onto it.

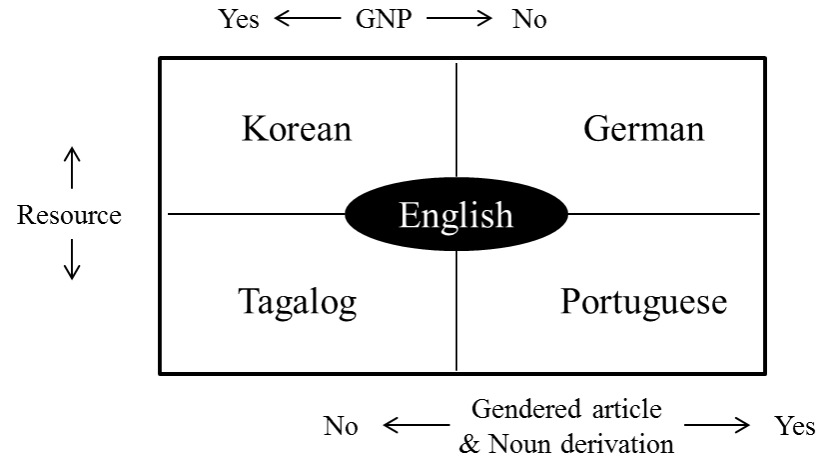

Figure 2: A simplified diagram for the typological approach regarding language selection. GNP exists in KO \& TL but not in DE \& PT; gender agreement/derivation in DE \& PT but not in KO \& TL. This framework will allow us to reflect on various types of gender bias in translators.

In this study, we focus on making up a more cross-lingually generalizable measure for evaluating the gender bias of MT systems as an application of NLP. To this end, we thoroughly sought how the previous approaches set up the measurements and target languages. There are two main aspects of the experiments performed depending on the type of language pair. One side measured the bias regarding the translation of gender-neutral pronouns, which exist in languages such as Korean, Japanese, or Turkish. In Prates et al. [20], a dozen languages were investigated with a template "The person is \#\#" via to-English translation, finding out that the output is tilted towards male-related expressions in the target language. The analysis was done with $p$-value, to show the biasedness of Google Translator ${ }^{1}$ for more than a thousand occupation words and about twenty adjectives. In Cho et al. [6], the authors suggested an advanced measure for the evaluation with occupation and sentiment words in Korean, and checked the validity of the measure when the answer is indeterminate due to the translation of gender-neutral pronouns being ambiguous.

The other side measured the bias in translation that brings up gendered articles. It was comprehensively investigated in Stanovsky et al. [25], using from-English translation into eight languages. In our study, the aforementioned types of analyses on translation gender bias are unified, to construct a prototype of a more crosslinguistically generalizable measurement.

\section{PROPOSED METHOD}

Our approach differs from the previous studies in that the multiple typological factors that affect the translation gender bias are taken into account. In the subsections below, the processes of language selection, template generation, and measure decision are described.

\subsection{Language Selection}

Here we present the grounds on which we decided the specific languages to utilize (Figure 2) and how the directions (KO-EN, TLEN, EN-DE, and EN-PT) are selected.

\footnotetext{
${ }^{1}$ https://translate.google.com/
} 
3.1.1 Languages of Interest. The languages of our interest here (hereafter referred to as LOI), namely German (DE), Korean (KO), Portuguese (PT), and Tagalog (TL), can be categorized both linguistically and concerning their publicly available language resources. The following three linguistic properties were crucial to our investigation: (a) presence of gender-neutral pronouns (GNP), (b) gender agreement of articles, and (c) noun derivation according to its gender.

(a) GNP is an umbrella term, defined here as a third-person singular pronoun that is not marked for gender distinction. Unlike English, where the GNP singular they is far from in general use ${ }^{2}$, Korean and Tagalog GNPs are frequently deployed in colloquial contexts [24, 28]. The presence or lack of GNP in specific languages can often reflect the algorithmic bias of translation services. For example, it can be observed in some translators that the Korean sentences "개는 경찰이야 ( $S / h e$ is a cop.)" and "개는 간호사야 $(S / h e$ is a nurse.)" are translated into English as "He is a cop." and "She is a nurse." respectively [6], where 개 (kyay) is a Korean GNP that can indicate either gender ${ }^{3}$. Similar holds for Tagalog siya.

(b) The agreement of the determiners with the gender of the head noun occurs in German and Portuguese but not in the other two LOIs [13, 24, 28, 32]. For example, when English "She is a cop." is translated into German as "Sie ist (eine) Polizistin.", the article $a$ in the source sentence turns into a gendered article eine, which agrees with the feminine noun Polizistin. This phenomenon sometimes induces errors such as "Sie ist ein Polizist.", on which the social bias regarding the occupation has an effect.

(c) Derivation of gendered nouns, where a gendered noun is assigned to a different gender by changing its form, often occurs in German and Portuguese [13, 32]. However, detecting errors in noun derivation can be tricky. In the aforementioned example, Polizistin is a feminine counterpart of masculine Polizist, whereas most loanwords (e.g., ein Barista, ein Model) or invariably gendered words (e.g., ein Genie; a genius) does not change according to the gender of the referent although grammatically correct. This factor affects the evaluation of the gender agreement of articles since machine translators quite often yielded invariable nouns or loanwords as the output.

Note that the concept of 'gender' utilized in (a) slightly differs with the one used in (b) and (c), since the gender of pronouns more regards the biological gender while the genderedness of articles and nouns is more grammatical. We may refer to the former when we deal with from-KO/TL translation, and the latter if the direction heads DE/PT, given that the errors in both types of gender mapping might cause a poor user experience.

3.1.2 Measure Language. Albeit we mainly analyze the languages above, the gap of grammatical genderedness between DE/PT and $\mathrm{KO} / \mathrm{TL}$ is considerable, that it is challenging to evaluate translation

\footnotetext{
${ }^{2}$ Although Bjorkman [3] suggests the recent shift of usage regarding singular they in modern English, it is not guaranteed that such tendency is well revealed in the corpora exploited in MT training phase so as to replace $s / h e$ in the real machine inferences.

${ }^{3}$ It must be noted that the neuter gender pronouns of German and Portuguese are distinguished from GNPs, and that the absence of GNP is not always equivalent with genderedness of the language. The latter is well illustrated with the example in the next subsection by contrasting English with German, a language with a full gender system.
}

\begin{tabular}{|c|c|c|c|c|}
\hline Languages & Documents & Sentences & Tokens (en) & Tokens \\
\hline German & 161,963 & $93.3 \mathrm{M}$ & $1.9 \mathrm{G}$ & $2.2 \mathrm{G}$ \\
\hline Portuguese & 162,430 & $77.7 \mathrm{M}$ & $1.5 \mathrm{G}$ & $1.4 \mathrm{G}$ \\
\hline Korean & 36,601 & $5.1 \mathrm{M}$ & $77.4 \mathrm{M}$ & $69.2 \mathrm{M}$ \\
\hline Tagalog & 17,031 & $1.3 \mathrm{M}$ & $36.3 \mathrm{M}$ & $26.9 \mathrm{M}$ \\
\hline
\end{tabular}

Table 1: The search result from OPUS.

gender bias using only the LOIs directly. Apart from LOIs, we need a language that is not the subject of analysis but used as the source or target language in the experiments. The following properties of English qualify it as a measure language.

First of all, although there exists 'singular they' in English, as described above, their appearance in MT inference is not significant to let English be considered as a language with GNP. This is relevant to examining errors in machine translation from $\mathrm{KO}$ or $\mathrm{TL}$, even when an overt antecedent is given, as in Figure 1.

Secondly, English does not have a gender system with an article agreement or active derivation of gendered nouns. In this way, the gender-related prejudice can be captured experimentally in EN-DE or EN-PT translation procedure.

3.1.3 Resource Issue. On top of the linguistic factors, we took the amount of available translation resources into consideration, since it is assumed to directly or indirectly affect the overall performance of (public) MT systems, either with training or pre-training [7, 12]. A recent article on the linguistic diversification [1] suggests that the resourcefulness in NLP is related to the amount of distributed usable libraries, models, and corpora, but it is difficult to quantitatively determine how under-resourced a language is.

For simple and effective comparison, we exploit the statistics of OPUS ${ }^{4}$, a repository of open MT datasets. The searched cases were EN-DE, EN-PT, KO-EN, and TL-EN. These include the number of documents, sentences, and tokens for English and the LOIs (as either target or source language).

One might claim that Portuguese is a high-resource language compared to Korean. However, here we intend to posit Portuguese and Tagalog as lower in resources than German and Korean, respectively (Figure 2), and compare the effect of the amount of resource on translator fairness between languages with similar grammatical properties regarding GNP or genderedness.

To add more on the measure language, English is dominating in the scale of publicly available resources [1] that adopting it as a measure language is less likely to affect the result regarding each language. Also, English is well supported in almost all translation services.

\subsection{Template}

In this section, we describe how we constructed the template sentences for the experiment. The general format is as:

One thing about the man/woman, [he/she] is [a \#\#] (inference) where [he/she] is either GNP or gender-specific terms depending on the language. is $[\boldsymbol{a} \# \#]$ refers to the predicate of the preceding

\footnotetext{
${ }^{4}$ http://opus.nlpl.eu/, as of October 2020.
} 
pronoun, which contains occupation or sentiment noun/adjectives, along with a copula and/or a nominative particle.

Further detail of the template: as shown in the previous section, the grammatical properties of a language largely influence the way that gender bias in translation is measured. Thus, we intended to create a sentence format that can render the projected gender bias relatively straightforward in the given types of languages. This is the background for the addition of a strong and straightforward context, One thing about the man/woman, which turns the task into a problem with an unambiguous answer. Previous works on languages with GNPs $[6,20]$ had limitations in this aspect, where there was no definite referent in the tasks. Although a less ambiguous approach was taken in Stanovsky et al. [25], the target languages were not without gender systems, and the template sentences involved indirect semantic cues rather than an overt expression of gender.

Another consideration was to make the template as independent as possible from sociocultural dependency. For example, in some of the previous studies on gender bias focusing on mono-lingual tendency [27, 31], we found it afraid to confirm that the adopted template can be utilized as well in multilingual settings, in the sense that they sometimes incorporate culture-specific contents. Therefore, by providing a strict condition that guides the coreference, we attempted to avoid inference depending on social context so that we can transparently measure the bias regarding gender, given a trained MT system. Though templates can reflect bias to varying degrees and the extent may depend upon language pair, we aimed to achieve reliable quantitative analysis by evaluating the accuracy of gender-related prediction.

\subsection{Measure}

As noted in Section 3.1, we identify three ${ }^{5}$ types of language by the existence of the gender system and gender-neutral pronouns. The first type is genderless languages with GNPs, such as Korean or Tagalog (Type 1). Another type displays agreement and derivation according to the grammatical gender, represented here by German or Portuguese (Type 2). The others are genderless languages with less dominance of conventional GNPs, viz. measure languages, to which English belongs. To disclose the gender bias, we could theoretically pair the languages in three ways: Type 1 to measure language, measure language to Type 2, and Type 1 to Type 2. In this paper, we only cover the first two ${ }^{6}$.

GNPs to non-GNPs: For the pairs Type 1 to measure language, i.e., $\mathrm{KO}$ or TL to EN, we checked if the translation of the genderneutral pronoun is in accordance with the information given in the preceding clause. As the task is relatively straightforward, we measured the accuracy, which approximately equals to the F1 score here. It should be noted that although evaluating gender bias in the translation of GNPs has been treated as describing a tendency rather than checking what is correct or not $[6,20]$, in this study, we regard it as a problem with an uncontroversial answer. Here, the

\footnotetext{
${ }^{5}$ In principle, there is yet another type of language, where there are both GNPs and a gender system, the most populous example being Hindi. We plan to explore such types of languages, but not at this point, mainly due to the problem formulation being challenging in translation.

${ }^{6}$ The last one, e.g., Korean to German, was omitted due to the difficulty in setting up the measure, possibly three or more factors, and will be investigated in future analysis.
}

content words are either names of occupation or sentiment words ${ }^{7}$ (e.g., gentle, sweet, greedy), as will be described below.

Agreement and derivation: For the pairs measure language to Type 2, i.e., EN to DE or PT, we first checked the gender of the translated lexical item. We mainly encountered three cases.

- When the lexical item is compatible with both genders (e.g., um/uma agente de leilões, an auction agent), we checked if the gendered article corresponds with the subject pronoun.

- When the gendered item has its counterpart in the other gender (e.g., um agricultor and uma agricultora, a farmer) we investigated if the gender of both the article and the noun matches with that of the subject.

- When the item is invariably assigned a single-gender as in anjo (angel in Portuguese), we made a tolerable judgment, e.g., both OK for ele é um anjo and ela é um anjo.

In this phase, the content words are solely comprised of nouns in the source sentences, i.e. names of occupations and sentimental prescriptions (e.g., fraud, pervert). However, if a non-noun or unseen expression emerges in the target languages, article agreement is checked upon the gender of the sentence subject. This is to be described in detail with the survey questions in the experiment section.

The fluency of translation: Besides gender-related measurements, we also checked whether the translation had been fluently performed. As to be described in the following section, we made up the gold standard for the template sentences and checked if the translation output matches with it, utilizing a conventional objective measure BLEU [19] and a recently proposed automatic semantic similarity checking system, BERTScore [29].

BLEU employs n-grams of the language to calculate the precision and brevity penalty within the predicted sentence. BERTScore adopts a large-scale unsupervised pre-trained language model [8] to gauge the pairwise cosine distance of the abstracted sentence representations. For the latter, one can claim that the approach incorporates existing biases into the pre-trained language model. However, our template sentences and resulting translation outputs are structured quite simple. Thus, we believe that the automatic evaluation methods would be free from the sentence style or nuance enough to judge only the semantic similarity of the predicted content word.

Miscellaneous: One may assert that severely flawed translations should not be counted as a valid test sample in checking the gender inferences. It might be reasonable to take into account only the cases the initial meaning is well preserved. Notwithstanding such concern, we investigated the whole output sentences because translation services primarily aim to assist people who are not familiar with either the source or the target language. In other words, gender specification occurring in the result is the first visible feature that can offend the user, perhaps more significantly than the fluency of the translation. In this regard, we divide between processes concerning gender assignment and fluency, and examine them separately.

\footnotetext{
${ }^{7}$ Sentiment words are adjectives in Type 1 to EN, while nouns in EN to Type 2. This will be explained in Section 4.
} 


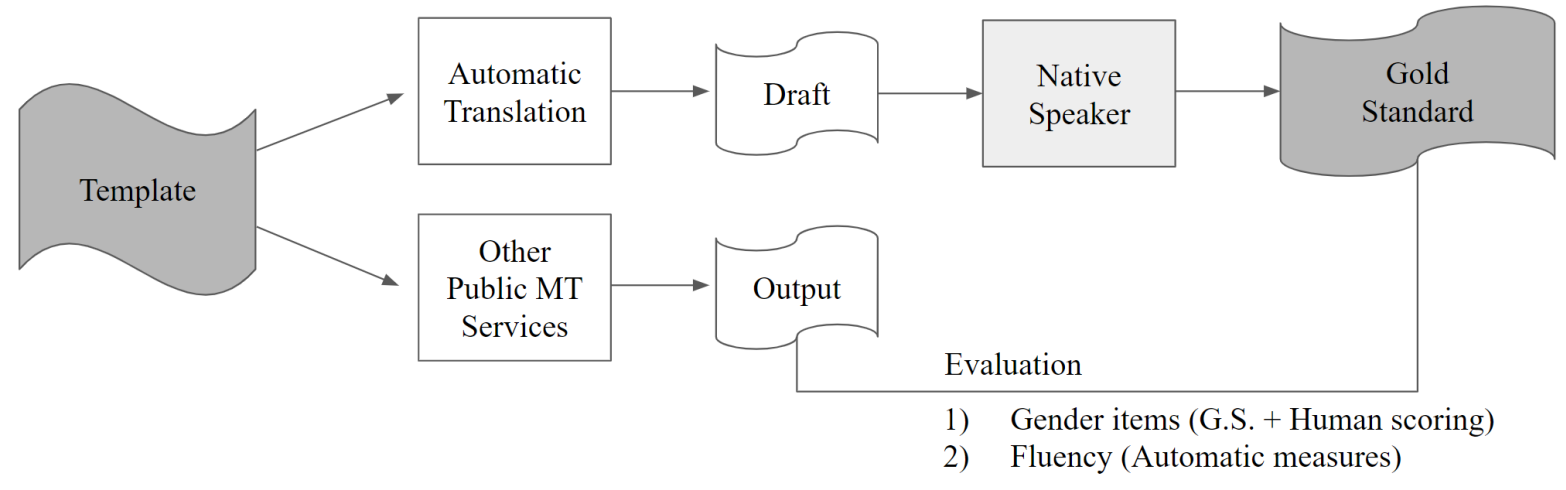

Figure 3: Flow chart for dataset construction and evaluation.

Another consideration is on the disparate impact, a measure to examine how the system performance of an underrepresented group, here the female, is not as significant as that of the other. This measure is not calculated in sentence-level, but after evaluating the whole corpus. We define it here as a female case accuracy divided by the male case accuracy, per each language pair.

\section{DATASET CONSTRUCTION}

We constructed gold standard sentences for all the languages used in the experiment, both the LOIs and the measure language.

\subsection{Content Words in Korean}

First, for the sake of public accessibility, we adopted the Korean content words (occupations and sentiment words) suggested in Cho et al. [6], and reexamined them to ensure that the phrases are not outdated in the spoken language. In this process, synonymous and related items were merged into one expression (e.g., home nurse and hospice nurse) so as to prevent the bias coming from the number of the branches. Also, only the expressions that are expected to be available cross-culturally were subject to consideration, although their validity may be further verified later in the translation phase. For that reason, three Korean native speakers searched and examined if the word is socially accepted (used with the public) or not.

Next, with the remaining content words, sentences were written in Korean in accordance with the template (Section 3.2). While doing so, the natives checked the naturalness of the expression in colloquial circumstances. The ones which failed to get consensus were removed or modified.

\subsection{Translation to English}

For the fluent and accurate translation to the other LOIs, we moved on to obtain a fairly reliable translation of the sentence set in the measure language. We achieved the translated version via the human and machine hybrid approach.

First, given the Korean template as the original, the initial English version was created with Google Translator, which was largely analyzed in the previous studies [6,20]. The output contained little amount of misinterpretations regarding gender and content, but was decent enough to be served as a draft translation that is independent with the tendency of the translators utilized in the validation phase ${ }^{8}$.

Consequently, four people who speak both Korean and English, including an English native, an L2 English speaker (Korean native), and two English learners (Korean native), checked the validity of the translation and only left the candidates that pass the felicity test. If the terms are dropped, the correspondings are simultaneously removed from the corpus of the other languages. This process is depicted in the top part of Figure 3.

The limitation here is that the sentiment words are merely prepared for the translation of Type 1 to measure language, as the LOIs of Type 2 sometimes may not show gender agreement for the translated terms of the adjectives. Thus, alternative sentiment nouns are required, especially those which prescribe the personality or behavior of people. We consulted the Senti-WordNet [9] for English sentiment words, and sorted out the nouns of which the gloss starts with either 'A person who' or 'Someone who'. Then we arranged the items by positivity and negativity scores, leaving only 230 of which either score is 0.25 or above. Terms that do not meet the ethical standard applied to sentiment words were removed referring to Cho et al. [6], that is, those related to appearance, economic status, sexuality, level of education, disability, etc.

\subsection{Translation to LOIs}

As a final step, we translated the English template sentences into the other LOIs, i.e. German, Portuguese, and Tagalog to get the gold standard. As in English, the draft MT output was post-edited by a native or at least bilingual speaker of each language, familiar with English and/or Korean. In specific, Tagalog native, Portuguese native, and L2 German speaker (Korean native), all native or expert in English, participated in making up the gold data.

In case the content word does not guarantee that it has consistent connotations inter-culturally, we asked the speakers to look carefully for that. For example, as military service is mandatory to most Korean males, the Korean language incorporates lots of widely used professions regarding military service: 'private first class', 'corporal', 'sergeant'. However, for a country like the Philippines, where the government policy differs from Korea, the job

\footnotetext{
${ }^{8}$ We do not use Google Translator in the experiment, since the translation fluency may be boosted in an unintended way for using it in the draft translation.
} 


\begin{tabular}{|c|c|c|c|c|}
\hline POS & Content & Volume & Template & Source \\
\hline Adj. & Sentiment & 68 & KO, TL & \multirow{2}{*}{ Cho et al. [6] } \\
\hline \multirow{2}{*}{ Noun } & Occupation & 187 & KO, TL, EN & \\
\cline { 2 - 5 } & Sentiment & 64 & EN & Senti-WordNet \\
\hline
\end{tabular}

Table 2: The composition of the final content words.

\begin{tabular}{|c|c|c|c|}
\hline Language Pair & \multicolumn{2}{|c|}{ Content } & Size of Gold Set \\
\hline KO/TL-EN & \multirow{2}{*}{ Occupation } & Senti. Adj. & $255 \times 2(\mathrm{EN})$ \\
\cline { 3 - 4 } & & Senti. Noun & $251 \times 2(\mathrm{DE} / \mathrm{PT})$ \\
\hline
\end{tabular}

Table 3: Language pairs of interest and the size of gold standard dataset. $\times 2$ denotes the augmentation by genderswapping.

market is not usually known for such levels. As a result, about 20 more content words were removed in the process ${ }^{9}$. Eventually, we achieved the complete list of the template sentences in all the languages, where $\mathrm{KO} / \mathrm{TL}$, EN serve as the source language (Table 2), and EN, DE/PT as the target language (Table 2, 3).

In summary, we obtained 187 nouns denoting occupations and 68 adjectives and 64 nouns concerning sentiments, which can be used flexibly depending on the language pair. This procedure is also described by the top part of Figure 3, only with the difference in the sentiment words of KO/TL-EN and EN-DE/PT (Table 3).

It is viable to claim that creating the template sentences from Korean and English might compromise the generalizability. However, it does not hinder our goals from building an inter-culturally applicable template, since all the involved languages undergo inspection regardless of the process being simultaneous or sequential ${ }^{10}$. By eliminating disputable terms and making the gold standard template vis-à-vis each language, we were able to extend the cross-linguistic utility of our equity evaluation corpus. All the data and further experiment results are to be shared in the public repository ${ }^{11}$.

\section{EXPERIMENT}

We studied three online translation services ${ }^{12}$, namely Yandex ${ }^{13}$, Microsoft Bing ${ }^{14}$, and Amazon AWS ${ }^{15}$, because they support all the LOIs, are freely available, and have numerous users worldwide. This follows the process depicted at the bottom of Figure 3. The other public MT module, which assisted us in obtaining the gold standard translations, was ruled out to guarantee a fair comparison.

As stated above, the primary measure is the accuracy of the prediction on the gender-related features, particularly the use of he or she for the Type 1 to measure language, and article agreement and noun derivation for the measure language to Type 2 pairs. We state a simplified guideline of the human evaluation below:

\footnotetext{
${ }^{9}$ The full sentence list is to be released with the paper.

${ }^{10}$ The weak point is that the initial lexicon pool concerns only Korean and English. However, we believe that the simultaneous creation of the profession list from all the languages would have also resulted in a similar outcome after the filtering.

${ }^{11}$ https://github.com/nolongerprejudice/tgbi- $\mathrm{x}$

${ }^{12}$ As of July 2020.

${ }^{13}$ https://translate.yandex.com/

${ }^{14}$ https://www.bing.com/translator

${ }^{15}$ console.aws.amazon.com/translate/
}

(1) Type $1(\mathrm{KO} / \mathrm{TL})$ to EN: One should check if the gender of the pronoun in the second clause, e.g., s/he or her/him, corresponds with the preceding. This does not necessitate the grammatical or semantic appropriateness of the output sentence.

(2) EN to Type 2 (DE/PT): It should be first checked that whether the translated gendered noun expression has been correctly derived.

- In case it matches with the gender of given pronoun (e.g., er or sie in German ${ }^{16}$ ), or is neutral, then one can check if the article agrees with the noun.

- In case not, one should check if the noun has the counterpart regarding gender. For example, Polizist (cop) in German has its counterpart Polizistin, while a loanword Model does not necessarily. If there exists, the noun derivation is decided wrong, and the article agreement is considered wrong as well. If no counterpart, we assume it correct, and the evaluation of the article agreement follows the noun.

(3) OK for no article: Some outputs in EN-PT translation showed no article. However, considering that in Portuguese, the article may be omitted under some conditions when the speaker does not want to judge the object, we annotated the result as correct. A similar holds for the occupation words in German.

For DE/PT, the total accuracy is counted only if both noun and article are appropriate.

The human evaluation of the gender-related factors was done with at least two bilinguals ${ }^{17}$, accompanied by the discussion with the authors, for all the language pairs. For the fluency check, we adopt corpus-level BLEU ${ }^{18}$ and BERTScore ${ }^{19}$. The total accuracy regarding human evaluation and fluency check are denoted as Total Acc. and Total Auto. in Table 4.

\subsection{Analysis}

The overall statistics suggest that accuracy is much lower for the female than the male, especially in $\mathrm{DE}$ and $\mathrm{PT}^{20}$, showing low disparate impact. Particularly, low disparate impact implies that the coreference might have been wrongly performed for some cases that require female inference. Also, the difference was displayed small between occupation and sentiment items in most cases, suggesting that the projection of bias is not restricted to social roles but also affects judgments on personality. Besides, there was little gap shown between the evaluated MT systems in total, while the details on each language pair differ upon the criteria. Beyond a mere numerical comparison of the MT modules and of the language pairs, below we provide a more detailed account of the results.

5.1.1 Per Occupation Group. One significant point is the stereotypes projected into the inference process. For example, in EN-DE, mistakes such as she is a game programmer to Sie ist ein professioneller Spieler, where ein and professioneller are both in malegendered, were commonly observed in all the translators. Also, in

\footnotetext{
${ }^{16} \mathrm{We}$ found no errors in the pronoun decision in DE/PT.

${ }^{17}$ For KO-EN and TL-EN, the biological gender of the inferred pronouns was checked. For EN-DE and EN-PT, two bilinguals each (at least with ten years of language experience for both languages) marked the machine inference.

${ }^{18}$ Smoothings were applied [5] and BLEU was scored using NLTK library [15].

${ }^{19}$ We utilized BERT multilingual, specifically bert-base-multilingual-cased, rescaled.

${ }^{20}$ Except for KO in Bing.
} 


\begin{tabular}{|c|c|c|c|c|c|c|c|c|c|c|c|c|}
\hline Systems & \multicolumn{4}{|c|}{ Yandex } & \multicolumn{4}{|c|}{ Bing } & \multicolumn{4}{|c|}{ AWS } \\
\hline \multirow{2}{*}{$\begin{array}{c}\text { Accuracy } \\
(\%)\end{array}$} & \multicolumn{2}{|c|}{ Noun / Article } & \multicolumn{2}{|c|}{ GNP } & \multicolumn{2}{|c|}{ Noun / Article } & \multicolumn{2}{|c|}{ GNP } & \multicolumn{2}{|c|}{ Noun / Article } & \multicolumn{2}{|c|}{ GNP } \\
\hline & DE & PT & KO & TL & DE & PT & KO & TL & DE & PT & KO & TL \\
\hline Occupation & $81.3 / 81.0$ & $98.7 / 97.9$ & 100 & 99.7 & $96.3 / 97.3$ & $96.8 / 95.7$ & 92.8 & 100 & $84.5 / 96.3$ & $98.1 / 98.1$ & 100 & 100 \\
\hline Sentiment & $85.9 / 80.5$ & $100 / 100$ & 100 & 100 & $95.3 / 90.6$ & $98.4 / 96.9$ & 69.9 & 100 & $89.1 / 84.4$ & $100 / 100$ & 100 & 100 \\
\hline Male & 99.6 / 98.8 & $100 / 99.2$ & 100 & 99.6 & $100 / 99.6$ & $98.8 / 97.2$ & 100 & 100 & 98.8 / 99.2 & $100 / 99.6$ & 100 & 100 \\
\hline Female & $65.3 / 62.9$ & $98.0 / 97.6$ & 100 & 100 & $92.0 / 91.6$ & $95.6 / 94.8$ & 73.3 & 100 & $72.5 / 87.3$ & $97.2 / 97.6$ & 100 & 100 \\
\hline Total Acc. & 80.3 & 98.4 & 100 & 99.8 & 94.4 & 95.2 & 86.65 & 100 & 82.9 & 98.2 & 100 & 100 \\
\hline D. I. & 0.625 & 0.980 & 1 & 1 & 0.896 & 0.960 & 0.733 & 1 & 0.690 & 0.972 & 1 & 1 \\
\hline BLEU & 68.4 & 55.0 & 32.1 & 40.6 & 75.7 & 41.6 & 32.1 & 34.1 & 43.7 & 50.1 & 35.0 & 32.9 \\
\hline BERTScore & 71.2 & 72.6 & 73.0 & 74.0 & 81.9 & 83.0 & 82.1 & 83.3 & 78.8 & 79.3 & 79.3 & 80.1 \\
\hline Total Auto. & 69.8 & 63.8 & 52.55 & 57.3 & 78.8 & 62.3 & 57.1 & 58.7 & 61.25 & 64.7 & 57.15 & 56.5 \\
\hline Per System & & $4.6 / 0.903 /$ & & & & $4.1 / 0.897 /$ & & & & $5.3 / 0.920 /$ & 9.9 & \\
\hline
\end{tabular}

Table 4: The evaluation result. Except for Per System, bold are the cases with the highest value. For Per System, Total Acc., disparate impact (D. I.), and Total Auto. are noted in the order.

EN-PT, some occupations were expressed in inappropriate feminine form as in lenhador (lumberjack) rather than lenhadora, which led to the use of um instead of uma. This was also the case for aviador, soldado, and monge (airman, soldier, monk), which should be aviadora, soldada, and monja. The opposite case includes uma babysitter for men.

In Type 1 to EN, where the errors rarely came up except for KO-EN (Bing), the bias was not manifested much. In Yandex, the only mistake found in TL-EN was the projection of male pronoun for guro sa elementarya (elementary school teacher), and no bias was shown in the content words in $\mathrm{KO}^{21}$.

Exceptionally, as for KO-EN in Bing, the pronouns were guessed almost randomly, especially for the female cases. The wrong answers include 경찰 (cop), 경비원 (guard), 잠수부 (diver), 배관공 (plumber), and some occupation group regarding programmer and developer, which are common gender stereotypical roles. We assume this phenomenon originated from the flaws in training KO-EN, e.g., wrong alignment of the tokens, which resulted in the nosedive of the total accuracy of the translator.

5.1.2 Per Sentiment Class. The proportion of errors in sentiment words was, in general as well, more significant in Type 2 languages than Type 1. Especially in EN-DE, for example a villain turned into ein Bösewicht for both genders in all the modules, and several more similar cases occurred. Also, in EN-DE/PT overall, it was exhibited that the errors mostly occurred in negative words, incorrectly inferring the woman as male. Besides the wrong coreferences, these results lower the disparate impact in general, as a consequence of the findings in Cho et al. [6] related to the bias regarding the gender appearance in the training corpus. On the other hand, we had no error regarding sentiment for KO/TL-EN in Yandex and AWS.

As stated above, the unexpected results came out with Bing, specifically for KO-EN. Although we experienced a malfunction, the tendency thereof was very helpful for the analysis regarding sentiment. It is straightforward that certain gender-stereotypical

\footnotetext{
${ }^{21}$ The insufficient translation fluency in Tagalog and Korean shown is mostly due to the absence of third-person singular pronouns in the output, not the incorrect inferences.
}

\begin{tabular}{|c|c|c|c|c|}
\hline Languages & DE & PT & KO & TL \\
\hline Unbiasedness & 85.8 & 97.2 & 95.5 & $\mathbf{9 9 . 9}$ \\
\hline D. I. & $\mathbf{0 . 7 3 7}$ & 0.971 & 0.911 & 1 \\
\hline BLEU & $\mathbf{6 2 . 6}$ & 48.9 & 33.1 & 35.9 \\
\hline BERTScore & 77.3 & 78.3 & 78.1 & $\mathbf{7 9 . 1}$ \\
\hline
\end{tabular}

Table 5: Aggregation of all the results of MT systems with respect to the languages. Unbiasedness denotes the total accuracy of the gender-related inferences.

items such as 약삭빨라 (weak ${ }^{22}$ ), 허약해 (fragile), or 귀여워 (cute) are likely to be correctly referred to as female, and that others such as 논리적이야 (logical), 용감해 (brave), 유치해 (childish), or 적극적이야 (active) are wrongly guessed as male.

These wrong coreferences not only lessens the quality of the translation but also reveals the social bias within the trained system. In addition, though the incorrectness in KO-EN or TL-EN seems more superficial than in those of EN-DE/PT, we deemed that the severity of the potential offense caused by the inaccuracy should not be underestimated for its simplicity.

5.1.3 Fluency and Biasedness. Beyond the numerics, we noted the relation between the translation fluency and the shown biasedness. At first, we suspected that the low translation fluency, which might be intertwined with the poverty of resources [12], would bring increased gender bias as a result. However, the high performance of translation fluency does not guarantee the same in bias issue. Comparing translation fluency and disparate impact within [DE vs. PT] and between [DE \& PT vs. KO \& TL] language types (Table 5), we learned that the MT systems with high translation fluency for some languages might also acquire the gender stereotypes on occupation or sentiment words, which is visible from the contrast of unbiasedness factors and fluency scores.

More on the fluency measures, we focused on the contrast of BLEU and BERTScore, which shows an inversed tendency overall

\footnotetext{
${ }^{22}$ The original meaning is more close to clever and weak is a mistranslation, but we leave it to the measurement of fluency and bypass these errors to focus more on the failure of gender coreference.
} 
(Table 5). It is also observed that BERTScore corresponds with the two gender bias measures, unlike BLEU that mainly concerns the token overlap of the prediction and the gold standard. This suggests that BERTScore can catch the gender-related aspects of the translation that BLEU might not get, though some kind of syntactic similarity can be overlooked.

Low-resourcedness. We took a further step to integrate the issue of semantic faithfulness of translation with the low-resourcedness. In general, the languages with more resources ${ }^{23}$ recorded better fluency index in to-English translation. Though indirectly, it can be a clue for a guess that large-scale resources boost the translation fluency but might make the system more biased.

The analyses above bases on the observation of the public MT models; thus, it is inevitable to face a missing link between the 'genuine' training resource and the shown biasedness. It is probable that for some language pairs, a data-efficient approach is working, while not for the others. Though we contrasted two factors unbiasedness and fluency in terms of language resource, the direct connection is not guaranteed.

Without a doubt, this might be well displayed if we train our own MT model, with controlled resources, and then compare the result as clearly as possible. However, it is unfortunate that the systems we train do not necessarily reflect the systems the MT users currently encounter. Moreover, such information on training resources is usually not provided by the service maintainers, making the indepth survey more challenging. In this regard, here we adhere to our original hypothesis that grounds on the survey over language resources, albeit the gap should be filled by further investigation.

\subsection{Discussion}

Upon the results, we hesitantly argue that the supplement of training resources may not be a solution for the mitigation of gender bias, but instead can be a trigger of the amplification. Although our observation cannot touch the core part of the training, the discussion is an extension of inductive bias in machine learning. Thus, further regularization is required to alleviate the bias, apart from collecting data and updating the performance.

One of such can be the detection of gender-specified or genderneutral context and modifying the related terms, as done in the recent approach of Google Translation ${ }^{24}$. In training-centered viewpoint, Saunders and Byrne [23] suggested a domain adaptation for the mitigation, by making up a small handcrafted set with a template "The [PROFESSION] finished [his|her] work" which gives the MT model a corresponding regularization in an additional training phase. The above works are concurrent with our approach, and we expect to extend our work, especially regarding language diversity and non-profession-related expressions, to real-world training. Notwithstanding such achievements, we raise two more issues here: English-centeredness and ethics.

Multilingualism. Despite the limitations that the languages investigated here do not and cannot fully represent the diverse human

\footnotetext{
${ }^{23}$ Although the information on public data (Table 1) has no direct link to the training procedure of the online MT systems, we hesitantly claim that the high translation fluency is partly supported by the amount of available MT resources.

${ }^{24}$ https://ai.googleblog.com/2020/04/a-scalable-approach-to-reducing-gender.html
}

languages, we believe that the direction of our research is to be beneficial for multilingual extension and generalization of the studies on translation gender bias. Though it is inevitably true that translation is a multilingual process, a large portion of the literature concentrated on measuring the bias in English, either as the source or the target. Here we attempted to shed more light on various properties of the languages on the other side, including morphosyntax, GNPs, low-resourcedness, and evaluation methodology. Nonetheless, we guess that the future research should incorporate the procedure of direct non-English-to-non-English translation, e.g., KO-DE, since the aspect of gender bias projected within would not be fully covered by the schemes proposed in this work and the previous studies.

Ethics. One may concern that there is no explicit discussion on ethics in this paper, either politically or philosophically. However, in the theoretical and sociolinguistic view, the struggle for deriving balancedness out of a biased system or data is itself a pursuit of ethical standards. Referring again to Friedman and Nissenbaum [11] and Binns [2], our approach is related to egalitarianism against data-driven biases, mainly regarding the areas of gender and multilingualism. For instance, our result was designed to imply the positive correlation between system performance and bias, not merely to evaluate the translation fluency. We further attempted to demonstrate that the systems trained with low-resourced languages generally have less (gender) bias-related errors compared to those of high-resourced ones.

We want to emphasize that we concentrated on the empirical perspective of fairness and transparency, although the underlying philosophy is obviously desired for a theoretical grounding, e.g., discrimination, egalitarianism, and justice [2]. We aimed to evoke both bias and cross-lingual issues by making up less controversial datasets, templates, and evaluation schemes.

\section{CONCLUSION}

In this paper, we argued for the stringent necessity of a crosslingual and sociolinguistic approach to measuring the gender bias in an inherently multilingual process: machine translation. Building on an equity evaluation corpus that incorporates inter-culturally present occupation and sentiment words, we chose the language pairs that highlight the various aspects of bias in gender-neutral pronouns, agreement of articles, and gendered noun derivation. The measurements, including the accuracy in gender-specific terms and the lexical-semantic similarity, were applied to three widely-used open-source MT modules.

We observed that there exists a trade-off between the amount of bias and translation fluency. The lower inference accuracy in the high-resource languages implicitly suggests the irony that the language resources could have provided the gender stereotype to the model being trained, thus calling for the algorithmic mitigation process to prevent possible errors that may offend anonymous users. To figure out the transparent cause and effect of bias, we could have built our model and found out factors. However, since such systems may not necessarily be used as a product, it would be challenging to estimate their influence on society. It would also be more meaningful to assess the bias within the publicly accessible models and in high demand, which are more likely to propagate and even amplify bias to NLP applications if adopted without inspection. 
As future work, we plan to explore different aspects of gender bias manifestation (e.g., gender agreement of other parts of speech, and other combinations of language pairs, not necessarily involving $\mathrm{EN}$ ), and to parlay our research into laying the foundation for an easily downloadable and deployable gender bias evaluation toolkit. All the templates and gold standard used in this research are to be released to the public ${ }^{25}$, and will be available for the service providers that deal with the gender-related errors of MT systems.

\section{ACKNOWLEDGMENTS}

The authors greatly thank Jihyung Moon and Sangwhan Moon for proofreading and giving important comments. Also, we highly appreciate the substantial feedback and thoughtful encouragement from all the anonymous reviewers. Most of all, we were fortunate to work with the great language specialists; Dong Sun Lim, Do Na Lee, Cheongwon Jang, Andreia Carvalho, Junwoo Song, Soohong Park, and Jieun Kim, without whom this research would have been impossible.

The open-access publication of this manuscript was sponsored by Technology Innovation Program (10076583, Development of free-running speech recognition technologies for embedded robot system) funded by the Ministry of Trade, Industry \& Energy (MOTIE, Korea).

\section{REFERENCES}

[1] Emily M Bender. 2019. The\# BenderRule: On Naming the Languages We Study and Why It Matters.

[2] Reuben Binns. 2018. Fairness in Machine Learning: Lessons from Political Philosophy. In Conference on Fairness, Accountability and Transparency. PMLR, 149-159.

[3] Bronwyn M Bjorkman. 2017. Singular they and the syntactic representation of gender in English. Glossa: a journal of general linguistics 2, 1 (2017), 80.

[4] Tolga Bolukbasi, Kai-Wei Chang, James Y Zou, Venkatesh Saligrama, and Adam T Kalai. 2016. Man is to Computer Programmer as Woman is to Homemaker? Debiasing Word Embeddings. In Advances in neural information processing systems. 4349-4357.

[5] Boxing Chen and Colin Cherry. 2014. A Systematic Comparison of Smoothing Techniques for Sentence-Level BLEU. In Proceedings of the Ninth Workshop on Statistical Machine Translation. 362-367.

[6] Won Ik Cho, Ji Won Kim, Seok Min Kim, and Nam Soo Kim. 2019. On Measuring Gender Bias in Translation of Gender-neutral Pronouns. In Proceedings of the First Workshop on Gender Bias in Natural Language Processing. 173-181.

[7] Stephane Clinchant, Kweon Woo Jung, and Vassilina Nikoulina. 2019. On the use of BERT for Neural Machine Translation. In Proceedings of the 3rd Workshop on Neural Generation and Translation. 108-117.

[8] Jacob Devlin, Ming-Wei Chang, Kenton Lee, and Kristina Toutanova. 2019. BERT: Pre-training of Deep Bidirectional Transformers for Language Understanding. In Proceedings of the 2019 Conference of the North American Chapter of the Association for Computational Linguistics: Human Language Technologies, Volume 1 (Long and Short Papers). 4171-4186.

[9] Andrea Esuli and Fabrizio Sebastiani. 2006. Sentiwordnet: A Publicly Available Lexical Resource for Opinion Mining. In LREC, Vol. 6. Citeseer, 417-422.

[10] Joel Escudé Font and Marta R Costa-jussà. 2019. Equalizing Gender Bias in Neural Machine Translation with Word Embeddings Techniques. In Proceedings of the First Workshop on Gender Bias in Natural Language Processing. 147-154.

[11] Batya Friedman and Helen Nissenbaum. 1996. Bias in Computer Systems. ACM Transactions on Information Systems (TOIS) 14, 3 (1996), 330-347.

[12] Joel Hestness, Sharan Narang, Newsha Ardalani, Gregory Diamos, Heewoo Jun, Hassan Kianinejad, Md Patwary, Mostofa Ali, Yang Yang, and Yanqi Zhou. 2017. Deep Learning Scaling is Predictable, Empirically. arXiv preprint arXiv:1712.00409 (2017).

[13] Amélia P Hutchinson and Janet Lloyd. 2003. Portuguese: An essential grammar. Psychology Press.

[14] James Kuczmarski and Melvin Johnson. 2018. Gender-Aware Natural Language Translation. (2018).

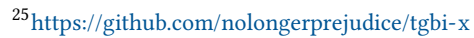

[15] Edward Loper and Steven Bird. 2002. NLTK: The Natural Language Toolkit. In Proceedings of the ACL-02 Workshop on Effective Tools and Methodologies for Teaching Natural Language Processing and Computational Linguistics. 63-70.

[16] Kaiji Lu, Piotr Mardziel, Fangjing Wu, Preetam Amancharla, and Anupam Datta. 2020. Gender Bias in Neural Natural Language Processing. (2020), 189-202.

[17] Shachar Mirkin, Scott Nowson, Caroline Brun, and Julien Perez. 2015. Motivating Personality-Aware Machine Translation. In Proceedings of the 2015 Conference on Empirical Methods in Natural Language Processing. 1102-1108.

[18] Malvina Nissim, Rik van Noord, and Rob van der Goot. 2020. Fair Is Better than Sensational: Man Is to Doctor as Woman Is to Doctor. Computational Linguistics 46, 2 (June 2020), 487-497. https://doi.org/10.1162/coli_a_00379

[19] Kishore Papineni, Salim Roukos, Todd Ward, and Wei-Jing Zhu. 2002. BLEU: A Method for Automatic Evaluation of Machine Translation. In Proceedings of the 40th annual meeting on association for computational linguistics. Association for Computational Linguistics, 311-318.

[20] Marcelo OR Prates, Pedro H Avelar, and Luís C Lamb. 2018. Assessing Gender Bias in Machine Translation: A Case Study with Google Translate. Neural Computing and Applications (2018), 1-19.

[21] Yusu Qian, Urwa Muaz, Ben Zhang, and Jae Won Hyun. 2019. Reducing Gender Bias in Word-Level Language Models with a Gender-Equalizing Loss Function. In Proceedings of the 57th Annual Meeting of the Association for Computational Linguistics: Student Research Workshop. 223-228.

[22] Ella Rabinovich, Raj Nath Patel, Shachar Mirkin, Lucia Specia, and Shuly Wintner. 2017. Personalized Machine Translation: Preserving Original Author Traits. In Proceedings of the 15th Conference of the European Chapter of the Association for Computational Linguistics: Volume 1, Long Papers. 1074-1084.

[23] Danielle Saunders and Bill Byrne. 2020. Reducing Gender Bias in Neural Machine Translation as a Domain Adaptation Problem. In Proceedings of the 58th Annual Meeting of the Association for Computational Linguistics. Association for Computational Linguistics, Online, 7724-7736. https://doi.org/10.18653/v1/2020.aclmain.690

[24] Paul Schachter and Fe T Otanes. 1983. Tagalog reference grammar. Univ of California Press.

[25] Gabriel Stanovsky, Noah A Smith, and Luke Zettlemoyer. 2019. Evaluating Gender Bias in Machine Translation. In Proceedings of the 57th Annual Meeting of the Association for Computational Linguistics. 1679-1684.

[26] Eva Vanmassenhove, Christian Hardmeier, and Andy Way. 2018. Getting Gender Right in Neural Machine Translation. In Proceedings of the 2018 Conference on Empirical Methods in Natural Language Processing. 3003-3008.

[27] Kellie Webster, Marta Recasens, Vera Axelrod, and Jason Baldridge. 2018. Mind the GAP: A Balanced Corpus of Gendered Ambiguous Pronouns. Transactions of the Association for Computational Linguistics 6 (2018), 605-617.

[28] Jaehoon Yeon and Lucien Brown. 2019. Korean: A comprehensive grammar. Routledge.

[29] Tianyi Zhang, Varsha Kishore, Felix Wu, Kilian Q Weinberger, and Yoav Artzi. 2019. BERTScore: Evaluating Text Generation with BERT. arXiv preprint arXiv:1904.09675 (2019).

[30] Jieyu Zhao, Tianlu Wang, Mark Yatskar, Vicente Ordonez, and Kai-Wei Chang. 2017. Men Also Like Shopping: Reducing Gender Bias Amplification using Corpus-level Constraints. In Proceedings of the 2017 Conference on Empirical Methods in Natural Language Processing. 2979-2989.

[31] Jieyu Zhao, Tianlu Wang, Mark Yatskar, Vicente Ordonez, and Kai-Wei Chang. 2018. Gender Bias in Coreference Resolution: Evaluation and Debiasing Methods. In Proceedings of the 2018 Conference of the North American Chapter of the Association for Computational Linguistics: Human Language Technologies, Volume 2 (Short Papers). 15-20.

[32] Gisela Zifonun, Ludger Hoffmann, Ursula Brauße, Bruno Strecker, and Joachim Ballweg. 1997. Grammatik der deutschen Sprache. Vol. 1. Walter de Gruyter. 